\title{
Superfluidity of "dirty" indirect excitons in coupled quantum wells
}

\author{
Oleg L. Berman ${ }^{1}$, Yurii E. Lozovik ${ }^{2}$, David W. Snoke ${ }^{3}$, and Rob D. Coalson ${ }^{1}$ \\ ${ }^{1}$ Department of Chemistry, University of Pittsburgh, \\ Pittsburgh, PA 15260, USA \\ 2 Institute of Spectroscopy, Russian Academy of Sciences, \\ 142190 Troitsk, Moscow Region, Russia \\ ${ }^{3}$ Department of Physics and Astronomy, University of Pittsburgh, \\ 3941 O'Hara Street, Pittsburgh, PA 15260 USA
}

(Dated: November 16, 2018)

\begin{abstract}
The theory of what happens to a superfluid in a random field, known as the "dirty boson" problem, directly relates to a real experimental system presently under study by several groups, namely excitons in coupled semiconductor quantum wells. We consider the case of bosons in two dimensions in a random field, when the random field can be large compared to the repulsive exciton-exciton interaction energy, but is small compared to the exciton binding energy. The interaction between excitons is taken into account in the ladder approximation. The coherent potential approximation allows us to derive the exciton Green's function for a wide range of the random field strength, and in the weak-scattering limit CPA results in the second-order Born approximation. For quasi-twodimensional excitonic systems, the density of the superfluid component and the Kosterlitz-Thouless temperature of the superfluid phase transition are obtained, and are found to decrease as the random field increases.
\end{abstract}

PACS numbers: 71.35.Lk, 73.20.Mf, 73.21.Fg, 71.35.-y

Key words: coupled quantum wells, superfluidity, indirect excitons, Bose-Einstein condensation of excitons

Superfluidity in a system of spatially indirect excitons in coupled quantum wells (CQW) has been predicted by Lozovik and Yudson,,$\frac{1}{1}$ and several subsequent studies 2.3.4.5.6.7.8.9.10.11.12 have predicted that this should be manifested as persistent electric currents, quasi-Josephson phenomena and unusual properties in strong magnetic fields. In the past ten years, a number of experimental studies have focused on this goal 13.14.15.16.17.18.19.20 The coupled quantum well system is conceptually simple: negative electrons are trapped in a two-dimensional plane, while an equal number of positive holes is trapped in a parallel plane a distance $D$ away. One of the appeals of this system is that the electron and hole wavefunctions have very little overlap, so that the excitons can have very long lifetime ( $>100 \mathrm{~ns}$ ), and therefore they can be treated as metastable particles to which quasiequilibrium statistics apply. Also, when $D$ is large enough, the interactions between the excitons are entirely repulsive, so that the exciton gas is stable against collapse (see, e.g., Ref. 21 22]).

In general, the theory of these systems has neglected the role of disorder. In real experiments, however, disorder plays a major role, arising from impurities, boundary irregularities, and fluctuations of the alloy concentration of the epitaxial layers. Although the quality of quantum well structures has improved dramatically over the past decade, e.g., the inhomogeneous broadening linewidth of typical GaAs-based samples has been improved from around $20 \mathrm{meV}$ to less than $1 \mathrm{meV}, 13$ the disorder energy is still not negligible compared to other energies. At a typical exciton density of $10^{10} \mathrm{~cm}^{-2}$, the interaction energy of the excitons is approximately $4 \pi \hbar^{2} a n / M D \simeq 1$ meV ( $a$ being the characteristic radius of a $2 D$ exciton, $M$ the total mass, and $n$ the number density of excitons) 20 Typical thermal energies at liquid helium temperatures are $k_{B} T=0.2-2 \mathrm{meV}$. On the other hand, the typical disorder energy of $1 \mathrm{meV}$ is low compared to the typical exciton binding energy of $5 \mathrm{meV}$.

Earlier studies of disorder in exciton systems include theory of the transport properties of direct and indirect excitons and magnetoexcitons in random fields, 23 the influence of various random fields on excitonic and

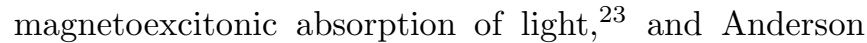
localization of excitons 24 In two-dimensional systems, the excitonic interaction in the Bogoliubov approximation is valid only at non-physically low densities because of the divergence of the two-dimensional scattering amplitude in the Born approximation 25 Therefore, the ladder approximation must be used at low densities to treat properly the interaction between two-dimensional excitons $\stackrel{25.26}{2}$ In this letter we study the case of a random field which is not necessarily small compared to the dipole-dipole repulsion between excitons. The coherent potential approximation 24 (CPA) allows us to derive the 2D indirect exciton Green's function for a wide range of the random field, and in the weak-scattering limit CPA results in the second-order Born approximation.

As mentioned above, fluctuations of the thickness of the quantum wells, which arise during the fabrication process, impurities in the system, and disorder in the alloy of the barriers can all lead to the appearance of a random field. Of these, spectral analysis of the exciton luminescence shows that alloy disorder, with a characteristic length scale short compared to the excitonic Bohr radius of around $100 \AA$, plays the most important role. In our model, the random potential $V(\mathbf{r})$ acting on an 
electron and hole is considered to be a Gaussian noise, such that

$$
\left\langle V(\mathbf{r}) V\left(\mathbf{r}^{\prime}\right)\right\rangle=g \delta\left(\mathbf{r}-\mathbf{r}^{\prime}\right), \quad\langle V(\mathbf{r})\rangle=0 .
$$

An electron is subjected to the potential $V_{e}=\alpha_{e} V(\mathbf{r})$ and a hole to $V_{h}=-\alpha_{h} V(\mathbf{r})$, where $\alpha_{e}$ and $\alpha_{h}$ are constants. We consider the characteristic length of the random field potential $l$ to be much smaller than the average distance between excitons $r_{s} \sim 1 / \sqrt{\pi n}(l \ll 1 / \sqrt{\pi n}$, where $n$ is the total exciton density). Therefore, in order to obtain the Green's function of the excitons with dipole-dipole repulsion in the random field, we obtain the Green's function of a single exciton in the random field (not interacting with other excitons), and then apply perturbation theory with respect to dipole-dipole repulsion between excitons, using the gas of non-interacting excitons as a reference system.

The momentum-frequency domain Green's function of the center of mass of the isolated exciton in the random field at $T=0$ within the coherent potential approximation $(\mathrm{CPA})$ is given by ${ }^{24}$ (here and below $\hbar=1$ )

$$
G^{(0)}(\mathbf{p}, \omega)=\frac{1}{\omega-\varepsilon_{0}(p)+\mu+i Q(\mathbf{p}, \omega)},
$$

where $\mu$ is the chemical potential of the system, and $\varepsilon_{0}(p)=p^{2} / 2 M$ is the spectrum of the center mass of the exciton in the "clean" system $\left(M=m_{e}+m_{h}\right.$ is the mass of the exciton; $m_{e}$ and $m_{h}$ are the electron and hole masses, correspondingly). The function $Q(\mathbf{p}, \omega)$ is determined by the effective random field acting on the center of mass of the exciton. For zero random field, $Q(\mathbf{p}, \omega) \rightarrow 0$. If $g \ll E_{b}$ ( $E_{b}$ is the binding energy of an exciton), the function $Q(\mathbf{p}, \omega)$ in the second-order Born approximation can be expressed through the ground-state wavefunction of an indirect exciton. Analytical results for $Q(\mathbf{p}, \omega)$ may be derived for $D \gg a$ and $m_{e}=m_{h}$ or $m_{h} \gg m_{e}$ and $\alpha_{h}\left(\frac{m_{h}}{m_{e}}\right)^{2} \ll \alpha_{e}$. We present in this Letter the simplest case $m_{e}=m_{h}$. The result, for the case $D \gg a$ and $m_{e}=m_{h}$, is

$$
\begin{aligned}
Q(\mathbf{p}, \omega)= & \frac{\left(\alpha_{e}-\alpha_{h}\right)^{2} M g}{16 \pi^{4}} \exp \left(-\frac{\rho^{2}}{8}\left(p^{2}+2 M \omega\right)\right) \\
& \times J_{0}\left(\frac{\rho^{2}}{4} \sqrt{2 M \omega} p\right),
\end{aligned}
$$

Here $\rho=(8 a)^{1 / 4} D^{3 / 4}$, with $a=\epsilon / 4 m_{e-h} e^{2}(\epsilon$ is the dielectric constant of the material, $m_{e-h}$ is the reduced mass of exciton, and $e$ is the electron charge), and $J_{0}(z)$ is a Bessel function of the first kind.

At small densities $n\left(n \rho^{2} \ll 1\right)$, the system of indirect excitons at low temperatures is a two-dimensional weakly nonideal Bose gas of dipoles with dipole moments d normal to wells $(d \sim e D)$. The distinction between excitons and bosons manifests itself in exchange effects (see, e.g., Refs. 21 22], and 27,28]). These effects are suppressed for excitons with spatially separated $e$ and $h$ in a dilute system $\left(n \rho^{2} \ll 1\right)$ at large $D(D \gg a)$, because at large $D$, the exchange interaction in the spatially separated system is suppressed relative to the $e-h$ system in a single well due to the smallness of the tunneling exponent $T \sim \exp \left[-(D / 2 a)^{1 / 4}\right]$ originating from the dipole-dipole interaction. Hence, when $D \gg a$, exchange phenomena, connected with the distinction between excitons and bosons, can be neglected, and therefore, the system of indirect excitons in CQWs can be treated by diagrammatic techniques developed for boson systems ${ }^{26}$. Two indirect excitons in a dilute system interact as $U(R)=e^{2} D^{2} /\left(\epsilon R^{3}\right)$, where $R$ is the distance between exciton dipoles along quantum well planes.

At the characteristic frequencies and momenta which give the greatest contribution to the Green's function in the ladder approximation, the function $Q(\mathbf{p}, \omega)$ can be approximated by the constant (see Eq. (3) $) Q(\mathbf{p}=\mathbf{0}, \omega=$ $0)=Q=\left(\alpha_{e}-\alpha_{h}\right)^{2} M g /\left(16 \pi^{4}\right)$. As a result, the first order approximation in the amplitude of scattering of the isolated pair of bosons $f_{0}$ the two-particle vertex $\Gamma$ can be reduced to $f_{0}$ pertinent to a clean system ${ }^{25.26}$, i.e. $\Gamma$ does not depend on $Q$ in the first approximation. As a result, the chemical potential $\mu$ has the same form as for the pure system ${ }^{25}$ :

$$
\mu=\frac{8 \pi n}{2 M \log \left(\frac{\epsilon^{2}}{8 \pi n M^{2} e^{4} D^{4}}\right)} .
$$

We have the condensate Green's function $D\left(\mathbf{p}, i \omega_{k}\right)$

$$
D^{(0)}\left(\mathbf{p}, i \omega_{k}\right)=\frac{-i(2 \pi)^{2} n_{0} \delta(\mathbf{p})}{i \omega_{k}+\mu+i Q},
$$

where $n_{0}$ is the density of Bose condensate. Since at small temperatures $\left(n-n_{0}\right) / n \ll 1$, according to the ladder approximation 26 we use $n$ instead of $n_{0}$ below. $G\left(\mathbf{p}, i \omega_{k}\right)$ and $F\left(\mathbf{p}, i \omega_{k}\right)$ are the normal and anomalous Green functions of the overcondensate:

$$
\begin{aligned}
& G\left(\mathbf{p}, i \omega_{k}\right)=-\frac{i \omega_{k}+\varepsilon_{0}(p)+\mu+i Q}{\omega_{k}^{2}+\varepsilon^{2}(p)-2 i\left(\mu-\varepsilon_{0}(p)\right) Q} ; \\
& F\left(\mathbf{p}, i \omega_{k}\right)=-\frac{\mu}{\omega_{k}^{2}+\varepsilon^{2}(p)-2 i\left(\mu-\varepsilon_{0}(p)\right) Q},
\end{aligned}
$$

where $\varepsilon_{0}(p)$ is the spectrum of noninteracting excitons; for small momenta $p \ll \mu$ the excitation spectrum of the interacting excitons, $\varepsilon(p)$, is acoustic: $\varepsilon(p)=c_{s} p$, where $c_{s}=\sqrt{\sqrt{\mu^{2}-Q^{2}} / M}$ is the velocity of sound.

We calculate the density of the normal component $n_{n}(T)$, using the Kubo formula ${ }^{29}$ and the total singleparticle Matsubara Green's function of the indirect excitons. We obtain

$$
n_{n}=n_{n}^{0}+\frac{N}{M} \int \frac{d \mathbf{p}}{(2 \pi)^{2}} p^{2} \mu \frac{\varepsilon_{0}(p)}{\varepsilon^{4}(p)} Q .
$$

Here $N$ is the total number of particles, and $n_{n}^{0}$ is the density of the normal component in a pure system with no impurities:

$$
n_{n}^{0}=-\frac{1}{2 M} \int \frac{d \mathbf{p}}{(2 \pi)^{2}} p^{2} \frac{\partial n_{0}(p)}{\partial \varepsilon} .
$$


where $n_{0}(p)=\left(e^{\varepsilon(p) / T}-1\right)^{-1}$ is the distribution of an ideal Bose gas of temperature excitations.

The first term in Eq. (7), which does not depend on $Q$, is the contribution to the normal component due to scattering of quasiparticles with an acoustic spectrum in an ordered system at $T \neq 0$. In a two-dimensional system,

$$
n_{n}^{0}=\frac{3 \zeta(3)}{2 \pi} \frac{T^{3}}{c_{s}^{4}(n, Q) M},
$$

where $\zeta(z)$ is the Riemann zeta function $(\zeta(3) \simeq 1.202)$. The second term in Eq. (7) is the contribution to the normal component due to the interaction of the particles (excitons) with the random field, which, when explicitly evaluated, yields:

$$
n_{n}=n_{n}^{0}+\frac{n Q}{2 M c_{s}^{2}(n, Q)} .
$$

The density of the superfluid component is $n_{s}=n-$ $n_{n}$. From Eqs. (9) and (10) we can see that increasing the random field decreases the density of the superfluid component.

In a $2 D$ system, superfluidity appears below the Kosterlitz-Thouless transition temperature $T_{c}=$ $\pi n_{s} /(2 M)^{30}$, where only coupled vortices are present. Using the expressions Eqs. (9) and (10) for the density $n_{s}$ of the superfluid component, we obtain an equation for the Kosterlitz-Thouless transition temperature $T_{c}$. Its solution is

$$
\begin{aligned}
T_{c} & =\left[\left(1+\sqrt{\frac{32}{27}\left(\frac{M T_{c}^{0}}{\pi n^{\prime}}\right)^{3}+1}\right)^{1 / 3}\right. \\
& \left.-\left(\sqrt{\frac{32}{27}\left(\frac{M T_{c}^{0}}{\pi n^{\prime}}\right)^{3}+1}-1\right)^{1 / 3}\right] \frac{T_{c}^{0}}{2^{1 / 3}} .
\end{aligned}
$$

Here $T_{c}^{0}$ is an auxiliary quantity, equal to the temperature at which the superfluid density vanishes in the mean-field approximation $n_{s}\left(T_{c}^{0}\right)=0$ :

$$
T_{c}^{0}=\left(\frac{2 \pi n^{\prime} c_{s}^{4} M}{3 \zeta(3)}\right)^{1 / 3}
$$

furthermore, in Eqs. (11) and (12),

$$
n^{\prime}=n-\frac{n Q}{2 M c_{s}^{2}} .
$$

The dependence of the Kosterlitz-Thouless transition temperature $T_{c}$ as a function of the total exciton density $n$ for different $Q$, obtained from Eq. (11), is presented in Fig. 1. It can be seen in Fig. 1 that the random field decreases the Kosterlitz-Thouless transition temperature. Fig. 2 shows the dependence of the KTS transition temperature on the random field parameter $Q$.

This work shows that although the random field depletes the condensate, Kosterlitz-Thouless superfluidity should still be possible in a system of spatially indirect excitons. For realistic experimental parameters taken from photoluminescence line broadening measurements ${ }^{13}$, the disorder energy is approximately $1 \mathrm{meV}$, which implies $Q=0.05$ in the units of the plots here, which implies a KTS transition temperature of $T=12 \mathrm{~K}$ at a density of $3 \times 10^{10} \mathrm{~cm}^{-2}$. This is still well below the density $n=1 / \rho(D)^{2}$ at which breakdown of the boson picture of excitons can occur, which for a typical electron-hole separation of $120 \AA$, i.e. $D=3$ in the units used here, is approximately $4 \times 10^{11} \mathrm{~cm}^{-2}$. On the other hand, for larger values of $Q$, which corresponds to sample quality of just a few years ago, the critical density can become quite close to this limit.

\section{Acknowledgements}

O. L. B. wishes to thank Prof. Dan Boyanovsky for many useful and stimulating discussions. Yu. E. L. was supported by the INTAS grant. D. W. S. and R. D. C. have been supported by grants from the National Science Foundation.
1 Yu. E. Lozovik and V. I. Yudson, Russ. JETP 44, 389 (1976).

2 Yu. E. Lozovik and A. V. Poushnov, Phys. Lett. A 228, 399 (1997).

3 Yu. E. Lozovik, I. V. Ovchinnikov, S. Yu. Volkov, L. V. Butov, and D. S. Chemla, Phys. Rev. B 65, 235304 (2002).

4 C. W. Lai, J. Zoch, A. C. Gossard, and D. S. Chemla, Science 303, 503 (2004).

${ }^{5}$ D. Yoshioka and A. H. MacDonald, J. Phys. Soc. of Japan, 59, 4211 (1990).

6 J. Zang, D. Schmeltzer and J. L. Birman, Phys. Rev. Lett.
71, 773 (1993).

7 Xu. Zhu, P. B. Littlewood, M. S. Hybertsen and T. M. Rice, Phys. Rev. Lett. 74, 1633 (1995).

8 S. Conti, G. Vignale and A. H. MacDonald, Phys. Rev. B 57, R6846 (1998).

${ }^{9}$ Yu. E. Lozovik, O. L. Berman and V. G. Tsvetus, Phys. Rev. B 59, 5627 (1999).

10 E. Demler, C. Nayak and S. Das Sarma, Phys. Rev. Lett. 86, 1853 (2001).

11 M. A. Olivares-Robles and S. E. Ulloa, Phys. Rev. B 64, 115302 (2001). 
12 S. A. Moskalenko, M. A. Liberman, D. W. Snoke and V. V. Botan, Phys. Rev. B 66, 245316 (2002).

13 D. Snoke, S. Denev, Y. Liu, L. Pfeiffer and K. West, Nature 418, 754 (2002).

14 D. Snoke, Science 298, 1368 (2002).

${ }^{15}$ L. V. Butov, C. W. Lai, A. L. Ivanov, A. C. Gossard, and D. S. Chemla, Nature 417, 47 (2002); L. V. Butov, A. C. Gossard, and D. S. Chemla, Nature 418, 751 (2002);

16 V. V.Krivolapchuk, E. S.Moskalenko, and A. L. Zhmodikov, Phys.Rev. B 64, 045313 (2001).

17 A. V. Larionov and V. B. Timofeev, Russ. JETP Lett. 73, 301 (2001).

18 J. A. Kash, M. Zachau, E. E. Mendez, J. M. Hong and T. Fukuzawa, Phys. Rev. Lett., 66, 2247 (1991).

19 U. Sivan, P. M. Solomon and H. Shtrikman, Phys. Rev. Lett. 68, 1196 (1992).

20 For a general review of experiments in quantum wells, see chapter 10 of S. A. Moskalenko and D. W. Snoke, BoseEinstein Condensation of Excitons and Biexcitons and Coherent Nonlinear Optics with Excitons (Cambridge University Press, New York, 2000).

${ }^{21}$ Yu. E. Lozovik and O. L. Berman, Russ. JETP 84, 1027 (1997).

22 Yu. E. Lozovik, O. L. Berman, and M. Willander, J. Phys.: Condens. Matter 14, 12457 (2002).

${ }^{23}$ Yu. E. Lozovik and A. M. Ruvinsky, Russ. JETP 87, 788 (1998).

24 Zh. S. Gevorkyan and Yu. E. Lozovik, Russ. Phys. Solid State 27, 1079 (1985).

${ }^{25}$ Yu. E. Lozovik and V. I. Yudson, Physica A 93, 493 (1978).

26 A. A. Abrikosov, L. P. Gorkov and I. E. Dzyaloshinski, Methods of Quantum Field Theory in Statistical Physics (Prentice-Hall, Englewood Cliffs. N.J., 1963).

27 B. I. Halperin and T. M. Rice, Solid State Phys. 21, 115 (1968).

28 L.V.Keldysh and A.N.Kozlov, Russ. JETP 27, 521 (1968).

29 G. D. Mahan, Many-Particle Physics, Plenum Press, New York (1990).

30 J. M. Kosterlitz and D. J. Thouless, J. Phys. C 6, 1181 (1973); D. R. Nelson and J. M. Kosterlitz, Phys. Rev. Lett. 39, 1201 (1977).

\section{Captures to Figures (1-2)}

Fig.1. Dependence of temperature of KosterlitzThouless transition $T_{c}=T_{c}(n)$ (in units of $R y *=e^{2} / \epsilon a$; $\left.a=\left(4 m_{e-h} e^{2} / \epsilon\right)^{-1}\right)$ on the exciton density $n$ (in units of $a^{-2}$ ) at the interwell distance $D=3$ (in units of $a$ ); at different random fields $Q$ (in units of $R y$ ): $Q=0$ - solid curve; $Q=0.05$ - dotted curve; $Q=0.1$ - dashed curve; $Q=0.2$ - dashed-dotted curve.

Fig.2. Dependence of temperature of KosterlitzThouless transition $T_{c}=T_{c}(Q)$ (in units of $R y *=e^{2} / \epsilon a$; $\left.a=\left(4 m_{e-h} e^{2} / \epsilon\right)^{-1}\right)$ on the random field $Q$ (in units of $R y$ ) at the interwell distance $D=3$ (in units of $R y$ ); at the different exciton densities $n: n=0.005$ - solid curve; $n=0.007$ - dotted curve; $n=0.01-$ dashed curve. 
Figure 1

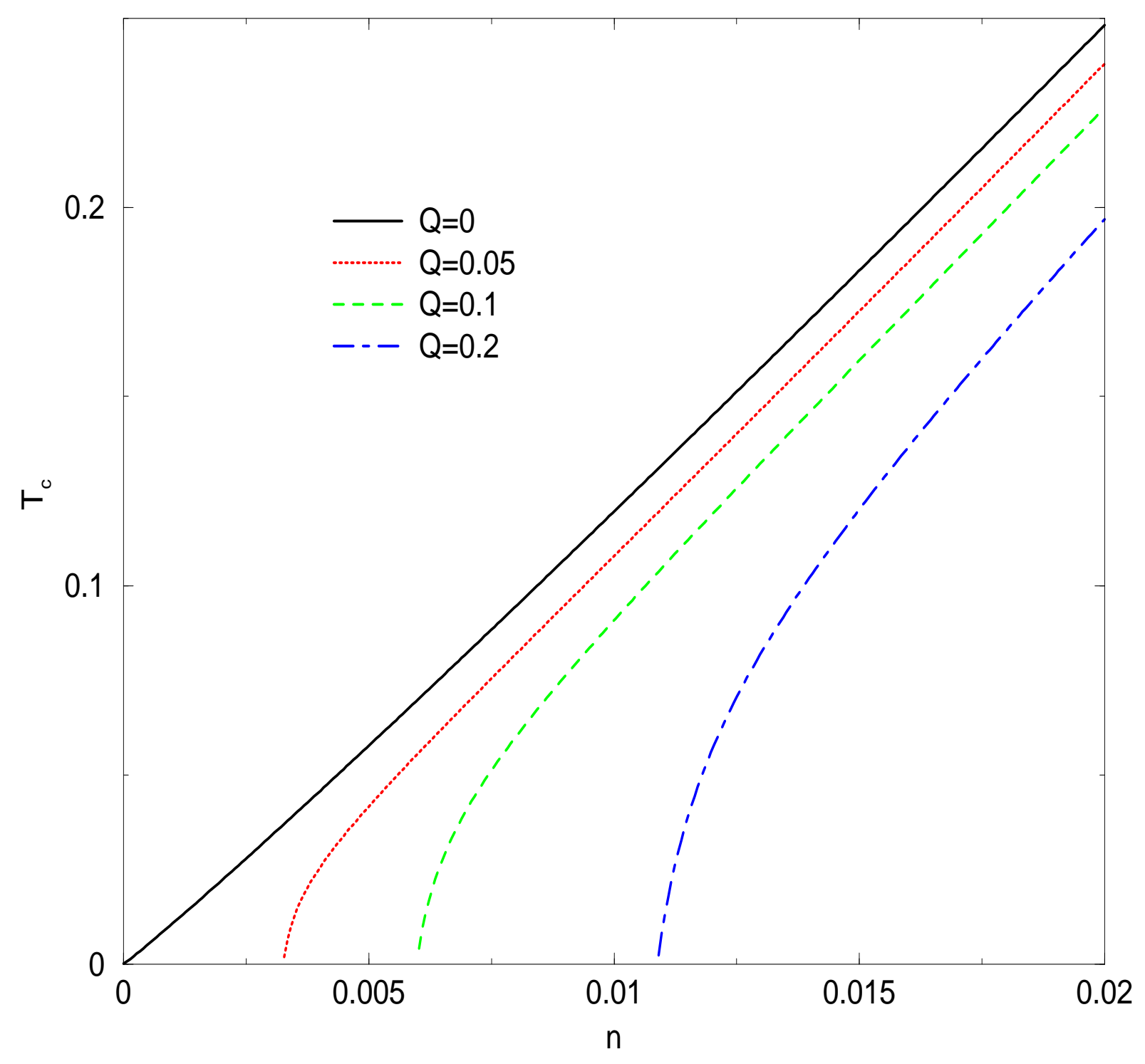


Figure 2

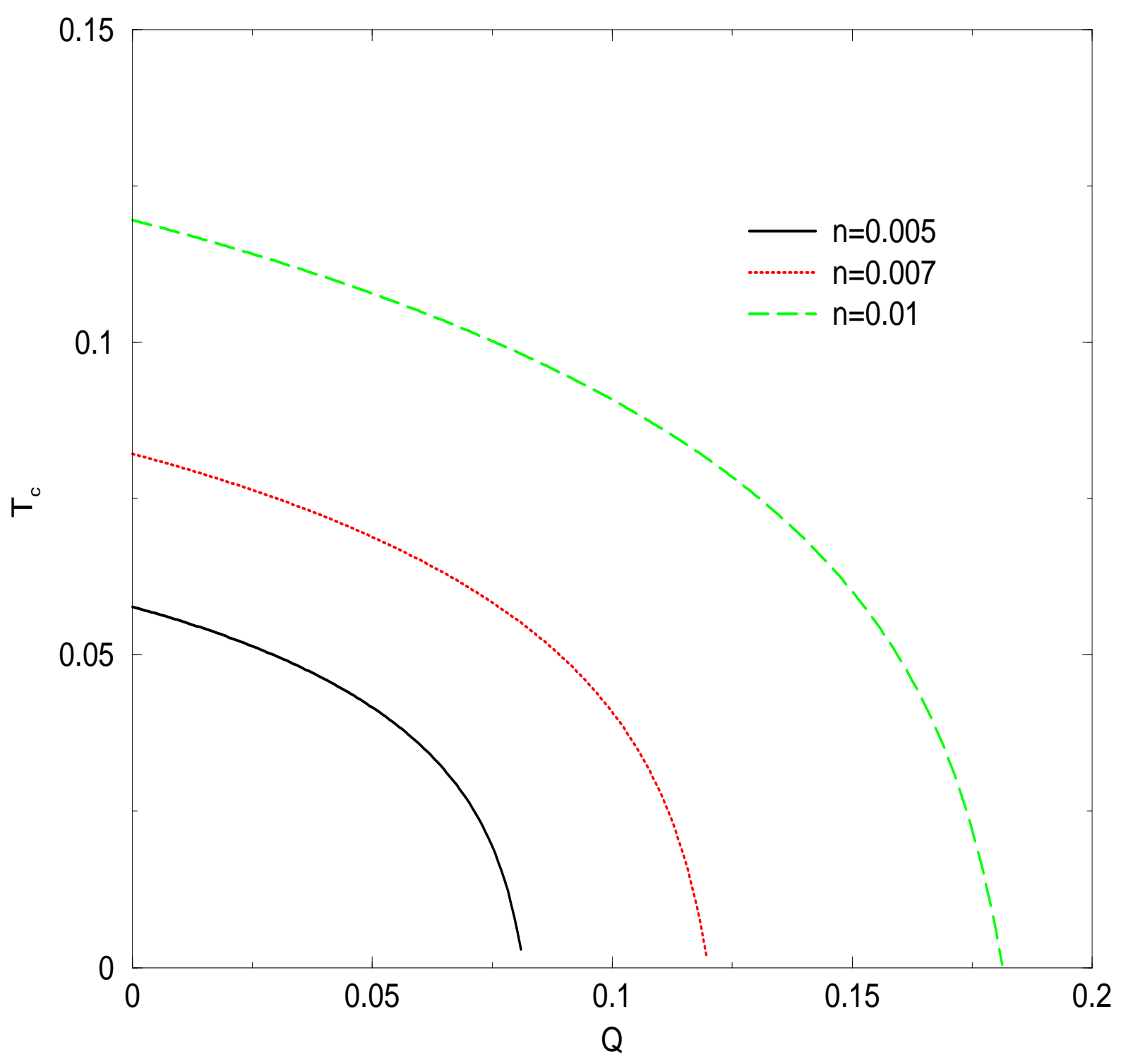

